\title{
ALCAM as a prognostic marker for acute ischemic stroke
}

A research team led by Thor Ueland of Oslo University Hospital in Norway reports that activated leukocyte cell adhesion molecule (ALCAM) could be a potential biomarker for early identification of patients at increased risk of mortality from acute ischemic stroke. "Patients with high levels of ALCAM at admission of acute ischemic stroke displayed increased long-term mortality," states Ueland. "[Our findings] suggest that ALCAM [is] a reliable biomarker for clinical use."
ALCAM is a proinflammatory cytokine involved in T-cell activation, stabilization of the immunological synapse and monocyte transendothelial migration-processes that have been implicated in acute ischemic stroke. In 244 patients aged $69 \pm 13$ years presenting with acute ischemic stroke, the researchers demonstrated increased levels of circulating ALCAM at admission, at days 1-5, and 6 months after stroke. Patients who died during a median of 4.4 years' follow-up were found to have

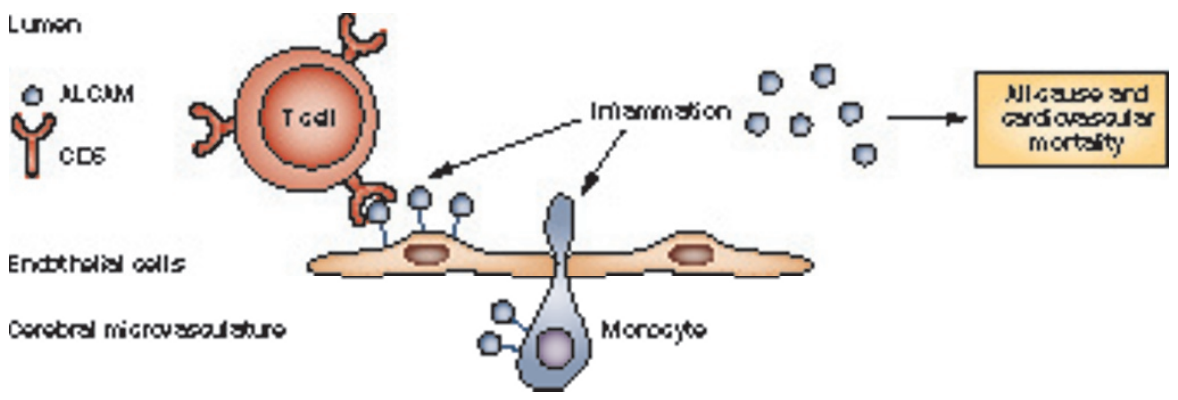

Enhanced inflammation may increase expression of ALCAM on endothelial cells and its receptor (CD6) on T cells, leading to enhanced T-cell activation and accelarated recruitment of T cells and monocytes into the cerebral microvasculature. These effects may be reflected by increased circulating soluble ALCAM and contribute to its utility in predicting adverse outcomes in stroke. Adopted from an image provided by Dr Thor Ueland.

higher levels of ALCAM at admission than survivors. Moreover, mortality (both all-cause and cardiovascular) was significantly increased in patients with ALCAM levels $\geq 46.8 \mathrm{ng} / \mathrm{ml}(P \leq 0.002)$, even when adjusted for other variables associated with mortality in ischemic stroke (such as stroke severity, cardiac troponin $\mathrm{T}$ and c-reactive protein levels, age, and prior heart and/or renal failure). These data suggest that high ALCAM levels $(\geq 46.8 \mathrm{ng} / \mathrm{ml}$ ) are an independent predictor of long-term mortality in patients presenting with stroke.

This study indicates that ALCAM may be an easily measurable and stable biomarker over the course of days after the index stroke event. Ueland and his team intend to investigate the inflammatory mechanisms involved in ALCAM's pathogenic role.

Iley Ozerlat

Original article Smedbakken, L. et al. Activated leukocyte cell adhesion molecule and prognosis in acute ischemic stroke. Stroke doi:10.1161/STROKEAHA.110.612440 